\title{
A Hierarchical Resource Allocation Algorithm for Satellite Networks Based on MF-TDMA
}

\author{
Huijun Feng ${ }^{1, a}$, Yuanan $\mathrm{Liu}^{2, \mathrm{~b}}$, Hefei Hu${ }^{2, \mathrm{c}}$, Dongming Yuan ${ }^{2, \mathrm{~d}}$ and Jing Ran², e \\ ${ }^{1}$ School of electronic Engineering, Posts and Telecommunications University, Beijing 100876, \\ China; \\ ${ }^{2}$ Posts and Telecommunications University, Beijing 100876, China. \\ a15210830135@163.com, byuliu@bupt.edu.cn, chuhefei@bupt.edu.cn, \\ dyuandm@bupt.edu.cn, ${ }^{\mathrm{e}}$ ranjing@bupt.edu.cn
}

Keywords: Satellite networks, hierarchical optimization, multi-priority services, big root pile, energy saving.

\begin{abstract}
Satellite communication networks would become one of the most prevalent communication system for the increasing demand for high-speed multimedia services and the underutilization of scarce satellite spectral resource. Considering resource optimization under multi-priority services and various link states in satellite networks, an optimal solution is sought and analyzed and a hierarchical resource allocation algorithm is proposed to minimize the energy consumption of the satellite links for given Quality of Service (QoS) requirements. In the hierarchical architecture, we propose a big root pile algorithm (BRPA) to select optimal slot collection, then a filling-water algorithm is utilized to allocate power resource among the chosen links. Numerical results validate the performance enhancement of the proposed scheme.
\end{abstract}

\section{Introduction}

Multi-frequency time division multiple access (MF-TDMA) is a multi-access scheme based on time and frequency division, which has been widely used in the satellite communication system [1, 3] For such a communication system, radio resource allocation is the core process of the ground-air-space integration [4]. Towards this direction, many interest of standardization groups and researchers has been focused on the extension of radio resource allocation and basic system models incorporating the wireless resource allocation have been presented in [5, 8]. These researches involves in power allocation, slot allocation and bandwidth allocation, as well as joint allocation strategy $[9,10]$.

However, these researches are mostly base on the ideal conditions. So fair resource allocation schemes for the satellite network should be investigated considering the realistic characteristics of both link-state and the different requirements of various kind of services. As shown in figure. 1, the different service requirements and link states are considered and a novel hierarchical slot and power allocation approach for the satellite networks is proposed. To consider the energy consumption of the satellite nodes, the conception of energy saving is employed [11], whereas a gain factor based on different priorities and link states are guaranteed through a specific network control center (NCC). Furthermore, in order to study a more realistic scenario, both the different service levels and link states are investigated. The performance of the proposed mechanism is evaluated through numerical simulation analysis.

\section{System Model}

In this section, we consider a hierarchical satellite networks architecture, where a network control center is introduced to control the process of the resource allocation. NCC is aware of the channel signal information (CSI) of each link and service level of each terminal through a spectrum manager. By collaborating with the satellite network [12], it involves in the allocation of slot and power. In 
MF-TDMA, satellite bandwidth available is divided into $M$ subcarriers, which share the same bandwidth and are available for the $\mathrm{K}$ terminals $(\mathrm{M}<\mathrm{K})$. While time is divided into different slots. In addition, it is assumed that terminals are capable to select optimal slots, but no more than one at a time. Therefore, each time slot allocated to a single terminal is contiguous in time with the same bandwidth.

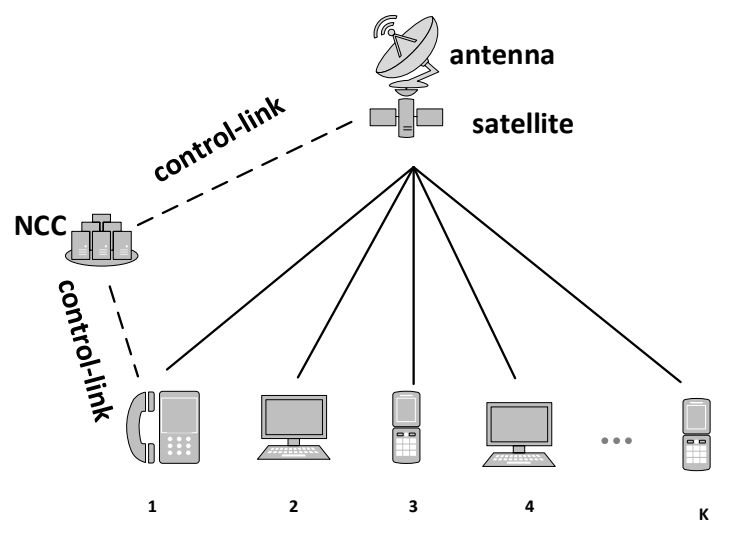

Fig. 1 Hierarchical access model

For guaranteeing the requirements of specific QoS, two functional levels have been considered: multi-priority services and different link states. The objective of the NCC is to minimize the energy of the satellite node, regardless of the cost between NCC and terminals as well as the satellite.

\section{Problem Formulation and Optimality Conditions}

\section{Problem Formulation.}

From Shannon capacity formula for Gaussian channel [13], the achievable data rate of $r_{i}$ of each terminal can be defined as $r_{i}=B^{\log \left(1+g_{i} p_{i}\right)}$. Therefore, the energy saving model can be formulated as (P1):

$$
\begin{aligned}
& \min _{s \in S_{s}} \mathrm{E}=\sum_{i=1}^{M} p_{s, i} t_{s, i} / \rho_{s, i} \\
& \text { S.t. } \quad \sum_{i=1}^{M} p_{i} \leq \mathrm{P} \\
& p_{s i \mathrm{i}} \geq 0 \quad \forall \mathrm{i} \\
& t_{s, i} \log \left(1+g_{s, i} p_{s, i}\right)=C_{s_{e} i} \quad \forall \mathrm{i} \\
& \mathrm{S}_{s}=\left\{\left(\partial_{s, 1}, \partial_{s, 2}, \partial_{s, 3}, \ldots \partial_{s, M}\right) \mid s=1,2,3, \ldots M !\right\}
\end{aligned}
$$

Where $t_{s, i}$ is the transmission time, ${ }^{P_{s, i}}$ is the power allocation for every terminal, $C_{s, i}$ is the capacity of a service, $P_{s_{2} i}$ is the utilization rate of the power and extra energy consumption caused by handover is mainly considered, the goal function is energy consumption.

\section{Optimality Conditions.}

To ensure that different service priorities, we employ the concept of gain factor $\partial_{k}$, which is defined as the maximum terminal gain that the channel can sustain for multi-priority services and different link states. In our analysis, the gain factor can be expressed analytically as:

$$
\partial_{k}=\lambda_{k} G^{T}\left|h_{k}\right|^{2} / B N_{0}
$$

Where $g_{k}=\left|h_{k}\right|^{2} / B N_{0}$ is the link transmission gain and is assumed flat during each transmission time interval, $\lambda_{k}$ is the different service levels, $G^{T}$ is the antenna gain of the satellite node. Considering each BW is the same, we take the problem of how to choose $\mathrm{M}$ terminals from $\mathrm{K}$ terminals $(\mathrm{M}<\mathrm{K})$ into account. For simplicity, the time is divided into different slots. We define each access-state collection as $\mathrm{S}_{s}$, so the system has total $\mathrm{s}(\mathrm{s}=\mathrm{K}$ !) states.

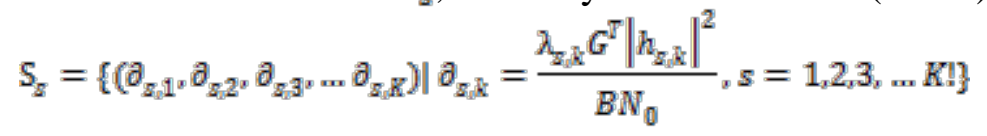


After calculating every gain factor using (1), the NCC chooses optimal access-state collection whose top $\mathrm{K}$ terminals will be selected to access the satellite network. Assuming the optimal access-state collection as $\mathrm{S}_{i}$.

$$
\left.\mathrm{S}_{i}=\left(\partial_{i, 1}, \partial_{i, 2}, \partial_{i, 3}, \ldots \partial_{i, K}\right) \mid \partial_{i, k}=\frac{\lambda_{i, k} G^{T}\left|h_{i, k}\right|^{2}}{B N_{0}}, \mathrm{i} \in\{1,2,3, \ldots \mathrm{K} !\}, \partial_{i, 1} \geq \partial_{i, 2} \geq \cdots \geq \partial_{i, K}\right\}
$$

Selecting top K terminals, the final slot collection is $S^{*}$.

$$
\left.S^{*}=\left(\partial_{1}, \partial_{2}, \partial_{3}, \ldots \partial_{M}\right) \mid \partial_{k}=\frac{\lambda_{k} G^{T}\left|h_{k}\right|^{2}}{B N_{0}}, \partial_{1} \geq \partial_{2} \geq \cdots \geq \partial_{M}\right\}
$$

Compared to the one for power and time allocation in orthogonal frequency division multiple access (OFDM) system, our formulation add an additional parameter $P$. Further, our system considers an optimal operation of gain factor, analyzes the multi-priority services and different link states, and proposes an efficient method to decide the optimal slot collections, which is unprecedented by any work.

\section{The Proposed Hierarchical Allocation Algorithm}

Note that the objective function in (1) is concave with respect to $\left\{\mathrm{S}_{s}, \mathrm{p}\right\}$, where a local maximum is also the global minimum [14]. What is more, the slot collection $\mathrm{S}_{\varepsilon}$ effects the optimization of the power resource. According to the proposed hierarchical allocation algorithm, the optimal slot collection S is selected via BRPA, then the power will be allocated according to the optimal slot collection.

\section{Big Root Pile Algorithm.}

A big root pile is a complete binary tree whose root is bigger than both left sub-tree and right sub-tree. When a new service coming or an old service completed, the NCC will select new slot collection based on a dynamic big root pile. Assuming that a node represents a unique service, the BRPA process is:
1. Calculate $\partial_{k}$ using (1).
2. Create a complete binary tree.
3. Adjust the binary tree into a big root pile.
4. If new service coming, go to 5

Else, go to 6.

5. Select the root node and put the last leaf node on the root.

6. Add the node to the last leaf node.

7. If a service is over, go to 8.

Else go back to 3.

8. Broadcast the slot collection to all the terminals.

Compared to direct plot selection, BRPA saves the last comparision result, thus having a higher efficiency.

Table 1 Time Complexity comparing

\begin{tabular}{|c|c|c|c|c|}
\hline Slot selection & Average & Best & Worst & Auxiliary Space \\
\hline Direct Selection & $\mathrm{O}\left(\mathrm{n}^{2}\right)$ & $\mathrm{O}(\mathrm{n})$ & $\mathrm{O}\left(\mathrm{n}^{2}\right)$ & $\mathrm{O}(1)$ \\
\hline BRPA & $\mathrm{O}\left(\mathrm{n} \log _{2}^{n}\right)$ & $\mathrm{O}\left(\mathrm{n} \log _{2}^{n}\right)$ & $\mathrm{O}\left(\mathrm{n} \log _{2}^{n}\right)$ & $\mathrm{O}(1)$ \\
\hline
\end{tabular}

\section{Power Allocation.}

According to the first step, the optimal slot collection $S^{*}$ is selected. That is to say, $\mathrm{M}$ terminals are selected to access the M subcarriers. Once a slot selected, it will never be free until the service is over, so the value of $\rho_{i}$ is 1 .Then the problem (P2) can be simplified:

$$
\min _{p i} \mathrm{E}=\sum_{i=1}^{M} p_{i} C_{i} / \log \left(1+g_{i} p_{i}\right)
$$

S.t. $\quad \sum_{i=1}^{M} p_{i} \leq \mathrm{P}$

$p_{i} \geq 0 \quad \forall \mathrm{i}$

For the optimal solution of problem P2, the Lagrange can be obtained as below. 


$$
\begin{aligned}
& \mathrm{L}\left(p_{i}, \mu_{i}\right)=\sum_{i=1}^{M} p_{i} C_{i} / \log \left(1+g_{i} p_{i}\right)+\mu_{i}\left(\sum_{i=1}^{M} p_{i}-P\right) \\
& \frac{\left.\partial \mathrm{L}\left(p_{i}, \mu_{i}\right)\right)}{\partial p_{i}} \begin{cases}=0, & p_{i}>0 \\
>0, & p_{i}=0\end{cases}
\end{aligned}
$$

Where shadow price $\mu_{\tilde{i}}$ is nonnegative Lagrange multiplier. By taking derivatives with respect to $\mu_{i}$ respectively, we can have the K-T conditions as, Assuming $f\left(p_{i}\right)=C_{i} / \log \left(1+g_{i} p_{i}\right)$

$$
\frac{\partial \mathrm{L}\left(p_{i}, \mu_{i}\right)}{\partial p_{i}}=p_{i} f^{\prime}\left(p_{i}\right)+f\left(p_{i}\right)+\mu_{i}
$$

Using (11) and (12), the relation between power and $\mu_{\tilde{i}}$ can be obtained

$$
p_{i}=\left(\frac{\mu_{i} f\left(p_{i}\right) \log s}{f\left(p_{i}\right)}-\frac{1}{g_{i}}\right)^{+}
$$

Where $(\mathrm{x})^{+}=\max \{\mathrm{x} \cdot 0\}$. For the optimal value of $p_{\tilde{i}}$, the well-known filling-water algorithm can be applied [15].

Based on the optimal conditions for the satellite network, we propose a hierarchical power and slot allocation algorithms. Note that the $p_{i}$ value is determined by the optimal slot collection. That is, the value of slot collection and $p_{i}$ is interactive. Although P1 could be solved via independent optimization technologies where power is allocation regardless of time slot, it is preferred to use a hierarchical optimization method in order to make a decision at each terminal under perfect slot collection. Hence, this algorithm shows possibility and feasibility of a hierarchical decision made by the NCC for the satellite network.

\section{Performance Evaluation.}

In this performance evaluation, we consider a simplified satellite network with multi-priority services based on MF-TDMA. It is assumed that the satellite network has total system bandwidth $100 \mathrm{Mbps}$, with the power available 200W. Typically, there are $8(\mathrm{~K}=8)$ acces points available to the satellite, which have different priorities $\boldsymbol{\lambda}_{\boldsymbol{k}}$. Furthermore, we suppose that CNR and different service levels $\lambda_{k}$ are random and follow a uniform distribution $\left(-20 \mathrm{~dB}<\boldsymbol{g}_{\boldsymbol{k}}<0 \mathrm{~dB}, 1<\boldsymbol{\lambda}_{\boldsymbol{k}}<10\right)$.

The average delay $\mathrm{T}=\sum_{i=1}^{M} \tau_{i}(T w+T t)$, where $T w$ is the waiting delay and the $T t$ is the transmission delay. $\tau_{i \mathfrak{i}}$ is the proportion of different priorities.

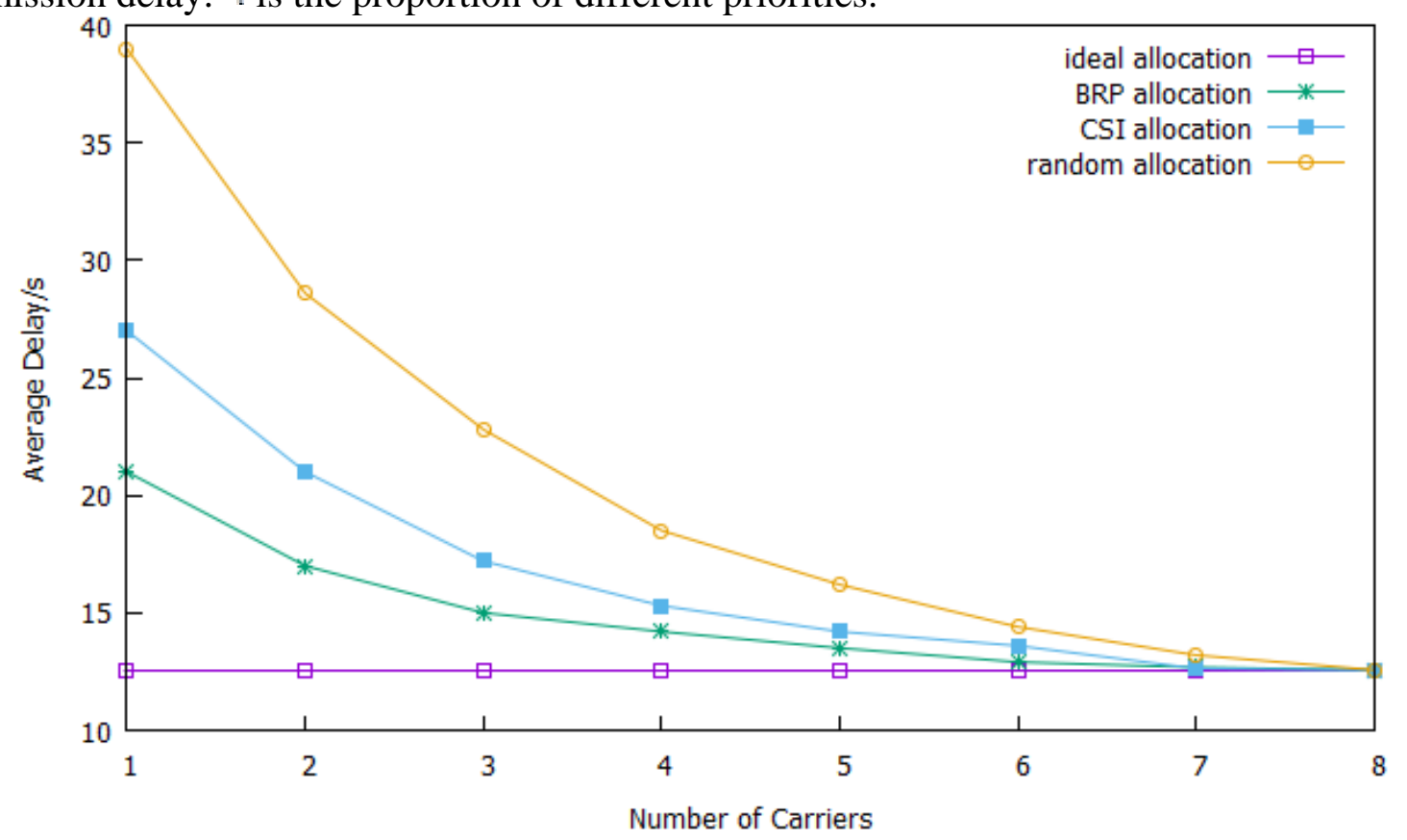

Fig. 2 Average delay of the service with four slot allocation methods.

Fig. 2 illustrate the average delay and the number of sub-carriers, for four slot allocation methods. They are ideal slot, BRPA, CSI and random allocation [16]. On the one hand, to some extent, the more the number of sub-carriers have, the less the average delay are. It is because an increase of 
sub-carriers will help reduce ${ }^{T w}$, thus contributing to decreasing average delay. On the other hand, random slot allocation has not considered the acces priority as well as the link state, so it has the biggest average delay. What is more, it can be seen that CSI allocation achies more average delay than BRPA, the reason is that it selects time slot only according to channel signal information $\boldsymbol{g}_{\boldsymbol{k}}$, whereas $\tau_{i \mathrm{i}}$ will influent the average delay. Hence, it can be asserted that by using BRPA scheme, the satellite network can satisfy various requirements of QoS with least time delay.

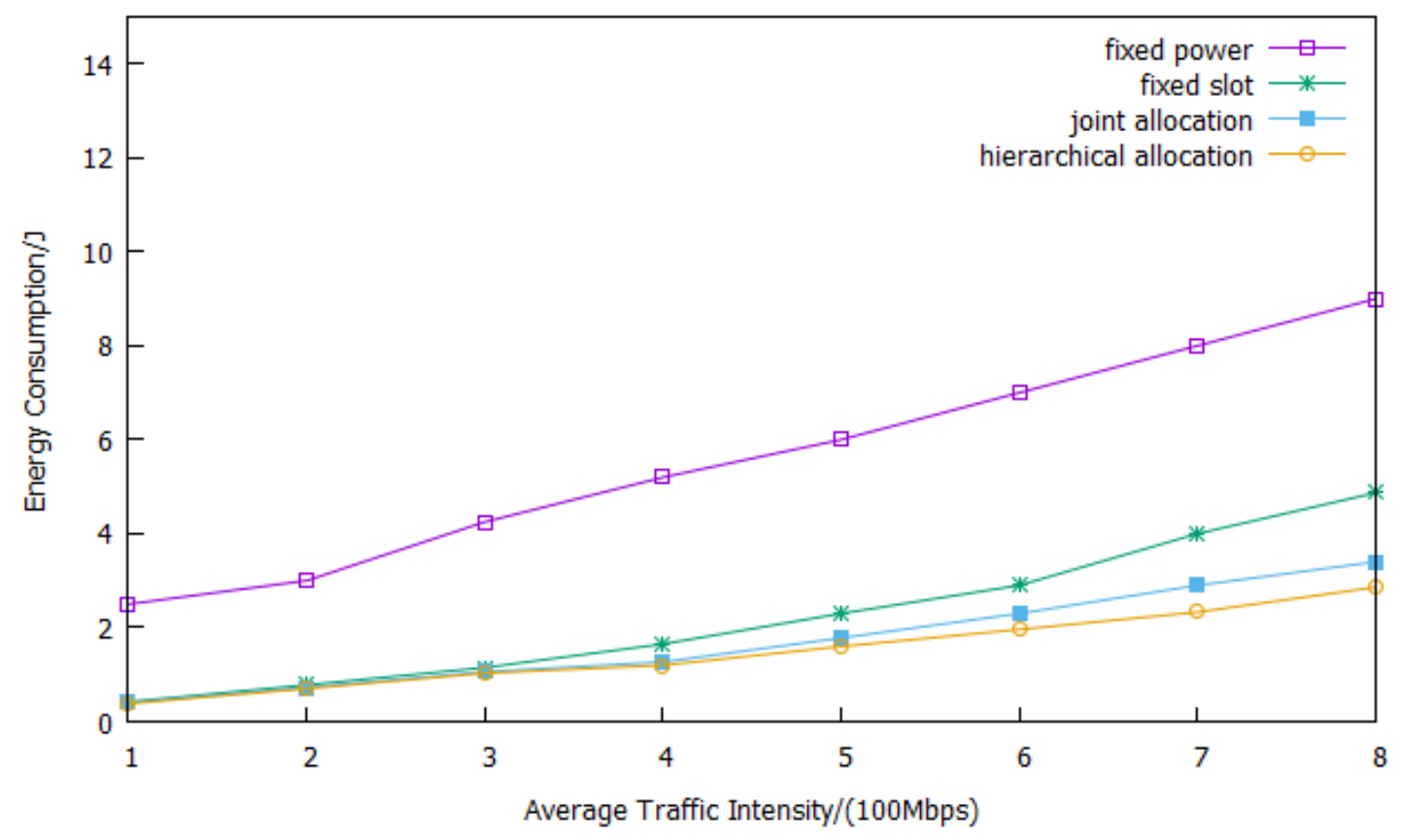

Fig. 3 Energy consumption with various traffic intensity

An example for finding an optimal solution for energy consumption is provided in Fig. 3. Particularly, in a satellite system based on MF-TDMA with $\mathrm{K}=4$, we apply the proposed hierarchical allocation for evaluation. the energy consumption is $\mathrm{E}=\sum_{i=1}^{M} p_{i} t_{i} / \rho_{i}$.

In Fig. 3, it is shown that the energy consumption increases as the average traffic intensity increases. These figures prove that fixed power allocation has the biggest energy consumption, then is the fixed slot allocation. With a consideration of extra energy consumption caused by handover, the hierarchical allocation has a higher utilization than joint allocation, especially in a higher traffic intensity. So the hierarchical allocation algorithm we proposed has a great potential in energy saving.

\section{Summary}

We analyzed the optical solution for satellite networks and proposed a hierarchical allocation algorithm for different requirements of QoS.Considering the multi-priority services and different link states, a novel time slot selection mechanism BRPA has been presented. Evaluation results based on the proposed scheme were shown that the hierarchical allocation was superior in average delay and energy consumption. A well-thought-out optimal solution in satellite with the diversity of complex business remains for future work.

\section{References}

[1]. Gan Z M, Zhang G X. Current development of satellite communication technology. Journal on Communications. Vol. 27 (2006) No. 8, p. 2-9.

[2]. Sara A, Eitan A, Jerome G, et al. Quasi-optimal resource allocation in multi-spot MF-TDMA satellite networks. Combinatorial Optimization in Communication Networks. Vol. 12 (2006), p. 325-366. 
[3]. Hao Jin , Yuan You , Xiaodong Ji , Yong Li , Wenbo Wang . An efficient network selection algorithm in integrated heterogeneous wireless network. IEEE Conference Publications, Nov. 2011,p. 511-516

[4]. Fabio Checconi, Luigi Rizzo, and Paolo Valente Efficient packet scheduling with tight guarantees. IEEE/ACM Transactions on Networking.Vol 21 ( 2013) No. 3,p. 802-816

[5]. Jie Miao, Hu Zheng, Yang Kun, et al. Joint Power and Bandwidth Allocation Algorithm with QoS Support in Heterogeneous Wireless Networks. Communications Letters, IEEE. Vol. 16 (2012) No. 4, p. 479-481.

[6]. He Li, Zeng Jie, Xin Su, et al. Joint Resource Allocation Method in Heterogeneous Wireless Networks Based on Genetic Algorithm. Wireless Communications \& Signal Processing. Huangshan, Oct. 2012, p. 1-5.

[7]. Hao Jin, You Yuan, Xiaodong Ji, et al. An efficient network selection algorithm in integrated heterogeneous wireless network. Broadband Network and Multimedia Technology (IC-BNMT). Shenzhen, Oct. 2011, p. 511-516.

[8]. Ismail M, Weihua Zhuang. Decentralized Radio Resource Allocation for Single-Network and Multi-Homing Services in Cooperative Heterogeneous Wireless Access Medium. Wireless Communications, IEEE. Vol. 11 (2012) No. 11, p. 4085-4095.

[9]. Gubong Lim, Cong Xiong, Cimini L J. Energy-Efficient Resource Allocation for OFDMA-Based Multi-RAT Networks. Wireless Communications, IEEE. Vol. 13 (2014) No. 5, p. 2696-2705.

[10]. Zheng G, Ma H, Cheng C, et al. Design and logical analysis on the access authentication scheme for satellite mobile communication networks. Information Security, IET. Vol. 6 (2010) No. 1, p. 6-13.

[11]. Guannan Xie , Huifang Chen , Lei Xie , Kuang Wang . A User-preference-aware Terminal-controlled Access Network Selection Scheme in Heterogeneous Wireless Networks. IEEE Conference Publications,Sep. 2013,p. 1-5

[12]. Jung M P, Savagaonkar U, Chong E K, et al. Allocation of QoS connections in MF-TDMA satellite systems. IEEE Transaction on Vehicular Technology. Vol. 54 (2005) No. 1, p. 178-192.

[13]. Yonghoon C, Hoon K, Sang-wook H, et al. Joint Resource Allocation for Parallel Multi-Radio Access in Heterogeneous Wireless Networks. Vol. 9 (2010) No, 11, p. 3324-3329

[14]. Yuan Sui, Ying Wei, Jinlong Zhang. Infant image registration based on improved mutual strict concave function measurement. Control and Decision Conference (CCDC). Qingdao, May. 2015, p.1123-1127.

[15]. Qilin Qi, Andrew Minturn, YaoQing Yang. An efficient water-filling algorithm for power allocation in OFDM-based cognitive radio systems. International Conference on Systems and Informatics. Yantai, May. 2012, p. 2069-2073.

[16]. Kawamoto Y, Fadlullah Z, Kato N, et al. Prospects and Challenges of Context-Aware Multimedia Content Delivery in Cooperative Satellite and Terrestrial Networks. IEEE Communication Magazine. Vol.52 (2014 ) No. 6, p. 55-61. 